\title{
Philonsorbonne
}

$7 \mid 2013$

Année 2012-2013

\section{"La grâce de l'esprit objectif ». Philosophie de la culture et ontologie de l'esprit chez Georg Simmel}

\section{Matthieu AMAT}

\section{(2) OpenEdition}

\section{Journals}

Édition électronique

URL : https://journals.openedition.org/philonsorbonne/469

DOI : 10.4000/philonsorbonne.469

ISSN : 2270-7336

Éditeur

Publications de la Sorbonne

\section{Édition imprimée}

Date de publication : 15 novembre 2013

Pagination : $9-26$

ISSN : 1255-183X

\section{Référence électronique}

Matthieu AMAT, « «La grâce de l'esprit objectif ». Philosophie de la culture et ontologie de l'esprit chez Georg Simmel », Philonsorbonne [En ligne], 7 | 2013, mis en ligne le 17 décembre 2013, consulté le 08 juin 2021. URL : http://journals.openedition.org/philonsorbonne/469 ; DOI : https://doi.org/10.4000/ philonsorbonne.469 


\title{
«La grâce de l'esprit objectif » \\ Philosophie de la culture et ontologie de l'esprit chez Georg Simmel
}

\author{
Matthieu Amat
}

L'expression «esprit objectif », utilisée hors du système de Hegel, sert généralement à désigner l'un des trois objets suivant: 1 . un ensemble de représentations collectives ou de manières d'agir ; 2 . un ensemble de réalités sur lesquelles l'esprit a déposé son empreinte et qui peuvent être considérées comme des objectivations de la vie spirituelle ; 3 . le mode d'être objectif de l'esprit par opposition à son mode d'être subjectif ou individuel. Le premier usage apparaitt dans un contexte sociologique au sens large, le second dans celui de la philosophie de l'histoire et/ou de la culture, le troisième relève de problèmes d'ontologie de l'esprit ${ }^{1}$.

Nous allons nous intéresser à la formation et à la fonction de ce concept chez Georg Simmel. La notion apparaît dans des développements qui relèvent ou bien du second, ou bien du troisième des contextes indiqués cidessus, ou bien encore des deux à la fois. Une élaboration particulièrement intéressante s'en trouve dans la seconde section du sixième chapitre de la Philosophie de l'argent, au croisement d'une analyse des processus historiques de la culture et de questions d'ontologie de l'esprit. L'objet de ce texte est $«$ la relation discordante entre culture objective et subjective $»^{2}$ : malgré le développement constant de la «culture des choses», celle des individus stagne ou même régresse. Si ce problème qui constituera le ressort de l'essai Le concept et la tragédie de la culture est relativement connu,

1. Pour le premier usage, voir par exemple R. Aron, Introduction à la philosophie de l'histoire, Paris, Gallimard, 1986, p. 87-94 et, plus récemment, V. Descombes, Les institutions du sens, Paris, Minuit, 1996, p. 267-308. Sur le second sens, voir W. Dilthey, L'édification $d u$ monde historique dans les sciences de l'esprit, Paris, Cerf, 1988, p. 100-105. Sur le troisième, voir H. Freyer, Theorie des objektiven Geistes, Leipzig-Berlin, Teubner, 1923, ou N. Hartmann, Das Problem des geistigen Seins, Berlin, de Gruyter, 1933.

2. G. Simmel, Philosophie des Geldes, in Georg Simmel Gesamtausgabe (désormais GSG), t. 6, Frankfurt am Main, Suhrkamp, 1989, p. 622 (désormais $P G$ ). Traduction française de S. Cornille et Ph. Ivernel : G. Simmel, Philosophie de l'argent, Paris, PUF, 1987, p. 575. 
son articulation avec le concept d'esprit objectif l'est beaucoup moins ${ }^{3}$. S'y intéresser, c'est se donner les moyens de préciser les fondements ontologiques de la philosophie de la culture de Simmel, souvent considérée comme une philosophie de la vie, mais dont nous voudrions montrer qu'elle est aussi une philosophie de l'esprit.

L' «esprit objectif» ne constitue pas chez Simmel une entité conceptuelle constamment distincte. Le lexique qui la désigne est varié. Dans le texte mentionné il est question d'esprit «objectif» (objektiv), «objectivé » (vergegenständlicht), «devenu objectif» (gegenständlich geworden), " réifié » (verdinglicht). Il en va de même pour ce qui concerne les concepts qui peuvent être traduits en français par «objectivation»: Vergegenständlichung, Objektivierung, Verdinglichung. Il s'agira de démêler ce qui tient du flottement sémantique - coutumier chez Simmel et ce qui relève de distinctions effectives.

Ces mises au point vont nous permettre de reconstruire ce qui paraitt constituer une intuition fondamentale à partir de la Philosophie de l'argent : il y a un primat ontologique, un excès d'être des contenus spirituels objectifs sur tout ce que les individus peuvent en tirer. Nous verrons que ce quasi a priori métaphysique se retrouve dans nombre de thèses de Simmel, à partir desquelles nous ferons valoir l'originalité de sa philosophie de la culture, à commencer par son irréductibilité à des modèles dialectiques ou expressivistes.

Les textes appelés en renfort de cette première mise au point sont le chapitre 6, section II de la Philosophie de l'argent (1900), le chapitre 2 des Problèmes fondamentaux de la philosophie ${ }^{4}$ (1910) et la conférence de 1918, De l'essence de la compréhension historique ${ }^{5}$.

\section{Esprit objectif et esprit objectivé}

\section{Une philosophie de la culture un peu convenue?}

La section de la Philosophie de l'argent qui nous intéresse a pour point de départ la distinction entre culture subjective et culture objective. Les

3. Il n'est fait par exemple aucune mention de Simmel dans les pages consacrées à l'esprit objectif dans l'Historisches Wörterbuch der Philosophie (J. Ritter, K. Gründer, G. Gabriel, Bâle, Schwabe Verlag, 1971-2005. Nous devons cependant indiquer l'article de Denis Thouard, Herméneutique et objectivité. Simmel et l'objectivité des sciences sociales et dire notre dette envers lui, notamment dans les développements finaux de ce travail. L'article est disponible en français sur le site halshs.archives-ouvertes.fr et a été publié en allemand dans l'Internationales Jahrbuch für Hermeneutik, 9, Tübingen, 2010.

4. G. Simmel, Hauptprobleme der Philosophie, in GSG 14 (désormais HP).

5. Id., Vom Wesen des historischen Verstehens, in GSG 16 (désormais VWHS). Traduction française de K. Winkelvoss : «De l'essence de la compréhension historique », in G. Simmel, La forme de l'histoire et autres essais, Paris, Gallimard, Le Promeneur, 2004. 
premières pages de la section définissent la «culture objective ». Celle-ci est présentée comme un «esprit objectivé» (gegenständlich gewordener Geist $)^{6}$, résultat du «travail d'innombrables générations ${ }^{7}$. En font partie langue, droit, religion, littérature, technique ; tous les faits que nous avons l'habitude de nommer culturels. Ce «travail spirituel condensé ${ }^{8}$ est une «objectivation de l'esprit »". Elle fait de l'homme un être historique car ayant un moyen d'assurer une « hérédité de l'acquis ${ }^{10}$, par laquelle en outre « un monde » ${ }^{11}$ lui est donné. Quant à la culture subjective, elle est la formation (Bildung) de l'individu rendue possible par la culture objective de son temps ${ }^{12}$.

Nous nous trouvons face à ce qui peut à première vue apparaitre comme une philosophie de la culture un peu convenue, reposant sur une théorie devenue commune de l'objectivation de l'esprit : la vie ayant atteint le stade de l'esprit s'extériorise, produit hors d'elle-même des réalités objectives. Dans un second temps, par un processus de «subjectivation», les individus ou les communautés peuvent jouir de ces contenus objectivés, se les réapproprier, et se cultiver à partir d'eux. Ne sommes-nous pas là face à une philosophie de la culture dialectique et expressiviste ? L'emploi régulier par Simmel d'un vocabulaire d'inspiration hégélienne ainsi que son inflexion progressive vers une «philosophie de la vie» paraissent justifier cette impression. Pourtant, si cette philosophie part de ces modèles, elle s'en départit ensuite et permet d'en penser les limites. C'est précisément le problème de la «relation discordante » entre culture subjective et culture objective qui va nécessiter leur dépassement et la production d'une philosophie de la culture sur d'autres bases - et notamment sur une nouvelle philosophie de l'esprit.

\section{Les deux sens d'« objectif »}

Nous devons clarifier le sens de l'adjectif - des adjectifs - qui qualifient l'esprit en tant qu'objectif. Nous pouvons distinguer deux sens d' « objectif ». L'adjectif peut désigner : 1. le fait pour une «chose » de faire face à un sujet (sens 1) - Simmel emploie en ce cas objektiv ou gegenständlich; 2. le fait, pour le contenu d'une représentation, pour une proposition, d'avoir une consistance propre qui lui donne un sens ne dépendant pas du point de vue de chaque sujet (sens 2) - Simmel emploie alors objektiv ou sachlich. Selon les textes, et à l'intérieur même de la Philosophie de l'argent, Simmel qualifie l'esprit d'objectif tantôt au sens 1,

\footnotetext{
6. G, p. 622 (trad. fr. p. 575).

7. Ibid., p. 622 (trad. fr. p. 574).

8. Ibid., p. 627 (trad. fr. p. 579).

9. Ibid. (Vergegenständlichung).

10. Ibid.

11. Ibid.

12. Ibid., p. 621 (trad. fr. p. 574).
} 
tantôt au sens 2 et utilise parfois le même mot (objektiv) dans les deux cas. Dans le premier cas, «esprit objectif » désigne un produit du travail humain en tant qu'il est un donné qui se tient face aux individus. En ce sens le tableau que je contemple est de l'esprit objectif. Dans le second sens, « esprit objectif » désigne ce qui dans le produit du travail humain a une signification indépendante du sujet, son contenu objectif. En ce sens aussi le tableau que je contemple est, ou plutôt contient, de l'esprit objectif. En nous autorisant d'un usage de Simmel - qui cependant n'est pas constant -, nous proposons de toujours parler d'esprit objectivé dans le premier cas (objectif au sens 1), d'esprit objectif dans le second (objectif au sens 2). Pour résumer, l'esprit objectivé est la culture objective, l'esprit objectif est la «signification idéelle » des objets de la culture objective ${ }^{13}$. En allemand, nous écririons objektiv seulement dans le second cas - ce que ne fait pas Simmel et utiliserions dans le premier cas - comme Simmel - gegenständlich geworden ou vergegenständlicht, ou encore objektiviert, selon un usage qui apparaît dans Le concept et la tragédie de la culture ${ }^{14}$.

\section{Culture objective et « royaume des idées » : ambiguïté et richesse d'une analogie}

\section{Le royaume des idées et la culture objective moderne : l'analyse du présent}

Après cette mise au point, voyons comment Simmel introduit ses concepts. Pour amener à penser la tension entre cultures objective et subjective, il convoque singulièrement le postulat platonicien de l'existence d'un «royaume idéal des valeurs théoriques $»^{15}$. La position de Platon est justifiée par le fait que tenir une pensée pour vraie implique de la concevoir comme «l'accomplissement d'une exigence objective, comme la reproduction d'un préfiguré idéel ${ }^{16}$. Contraint de donner un mode d'être à ce «préfiguré idéel », l'esprit conçoit alors un «royaume» qui «inclut toute vérité possible ${ }^{17}$. Or, la relation entre ce royaume idéal et l'individu aspirant à la connaissance :

a son analogue en dimension réduite dans la relation entre l'esprit et la culture objectivés (vergegenständlicht) d'une part, et le sujet individuel d'autre part ${ }^{18}$.

13. Ibid., p. 627 (trad. fr. p. 579).

14. Der Begriff und die Tragödie der Kultur, in GSG 14, p. 391 (désormais BTK). Traduction française de Ph. Ivernel : «Le concept et la tragédie de la culture », in La Tragédie de la culture, Paris, Rivages, 1988, p. 186.

15. $P G$, p. 623 (trad. fr. p. 576).

16. Ibid., p. 623 (trad. fr. p. 575).

17. Ibid., p. 623 (trad. fr. p. 576).

18. Ibid., p. 626 (trad. fr. p. 578). 
De même que sur le plan épistémologique l'individu doit se soumettre à des contenus qui ne dépendent pas de lui, si bien que la connaissance est une sorte de reproduction de contenus valides en soi, de même l'individu se cultivant fait face à « des contenus préformés, invitant à leur réalisation dans les esprits individuels ${ }^{19}$. Ces contenus constituent la culture objective propre à un lieu et à un temps. Ils sont donnés, hérités, l'individu se trouve face à eux qu'il le veuille ou non. Simmel invite à penser la culture objective comme un monde consistant et indépendant : il parlera dans Le concept et la tragédie de la culture de «cosmos autonome de l'esprit objectivé ${ }^{20}$. Le constat de la distance croissante entre individu et culture objective résulte de cette prémisse. Simmel ajoute plus loin :

La splendeur et la grandeur de la culture moderne présentent ainsi quelque analogie avec ce lumineux royaume des Idées chez Platon, où l'esprit objectif (objektiver Geist) des choses est là dans sa perfection immaculée ${ }^{21}$.

Cette précision est importante : aucune époque de la culture ne mérite autant que la culture moderne d'être comparée au "royaume des Idées ». Dans sa quantité et sa variété, par la longueur et la complétude de ses séries d'objets (objets techniques, théories et propositions scientifiques, textes juridiques, histoire des arts, etc.) et parce qu'elle tend à rendre disponible les œuvres de tous les temps et de tous les pays, la culture objective moderne s'apparente de plus en plus à un monde plein et consistant et, pour ainsi dire, à un royaume des Idées réalisé. Cette perfection objective a cependant pour revers de la rendre de plus en plus étrangère aux fins individuelles.

\section{Le royaume des idées et l'esprit objectif: au fondement de la philosophie de la culture}

Si l'analogie platonicienne de Simmel est stimulante, elle n'est pas sans ambiguïté. Elle renforce même le risque de confusion entre esprit objectif et esprit objectivé. En effet, le «royaume des Idées » en tant que «royaume de validités objectives ${ }^{22}$ ne tient-il pas plus de l'esprit objectif que de la culture objective? Les «contenus préformés » de culture face auxquels se tient l'individu ne désignent-ils pas les contenus idéels des objets culturels (de l'esprit objectif donc), et non ces objets culturels (esprit objectivé) ? Cela apparaît clairement lorsqu'on lit quelques lignes plus bas que ces contenus :

«conserv[ent] aussi leur détermination (Bestimmtheit) au-delà [des individus], sans qu'elle soit non plus celle d'un objet matériel; car même lorsque l'esprit est lié à des matériaux - outils, œuvres d'art, livres - il ne coïncide jamais avec ce qui, dans ces choses, tombe sous les sens $\gg^{23}$.

19. Ibid. (trad. modifiée).

20. BTK, p. 391 (trad. fr. p. 186).

21. $P G$, p. 649 (trad. fr. p. 598).

22. Ibid., p. 626 (trad. fr. p. 578).

23. Ibid., p. 626 (trad. fr. p. 578). 
Simmel prend bien soin de distinguer l'objet culturel et son contenu ou esprit. Il ne s'agit plus ici de décrire un état de la culture objective mais d'introduire à une ontologie de l'esprit. Dans un texte de 1910, Les Problèmes fondamentaux de la philosophie $e^{24}$, Simmel réutilise le modèle platonicien à cette fin. Dans le troisième chapitre, il affirme que Platon a découvert l'esprit objectif. Il utilise alors l'expression de «troisième royaume », par quoi Frege désignait le «lieu » des pensées avec leur sens intemporel, lieu irréductible aux mondes intérieurs (lieu des représentations) ou extérieurs (lieu des choses) ${ }^{25}$. Simmel dit toutefois la même chose que dans le passage que nous venons de citer : les contenus doivent être distincts à la fois de leurs supports matériels et de leur mode d'être dans le psychisme individuel. Ainsi l'esprit objectif peut-il être décrit comme "un royaume de contenus idéels qui n'est ni subjectif ni objectif ${ }^{26}$ (au sens 1). Simmel ajoute assez allusivement, que «l'être métaphysique » de ces contenus «se tient au-delà de la question de l'existence objective-extérieure ou subjectivepsychique, mais aussi au-delà de celle de l'être et du non-être en général $»^{27}$. Un mode d'être spécifique doit être accordé à l'esprit objectif, indépendant de l'alternative être/non-être, c'est-à-dire, selon Simmel, substance/néant. Platon a essayé de penser ce mode d'être, mais n'a pas su s'émanciper du concept de substance, prégnant dans la culture grecque. Simmel défend donc un certain réalisme sémantique, que l'on aimerait parfois plus précis mais qui détermine certains des caractères de sa philosophie de la culture.

La fonction de la référence à Platon dans les Problèmes est plus claire que dans la Philosophie de l'argent, où le sens de l'analogie est ambigu ambiguïté renforcée par le flottement sémantique entre esprit objectif et objectivé. En réalité, l'analogie a plusieurs fonctions, peut-être encore insuffisamment distinctes en 1900. D'une part elle vise à attirer l'attention sur la distance croissante entre les individus et la culture objective. D'autre part, elle invite à des considérations philosophiques fondamentales quant à l'être des contenus de la culture. Elle sert à décrire un état de la culture objective mais aussi à poser les jalons d'une ontologie de l'esprit ; elle est au service de l'analyse de la culture moderne et de la «crise de la modernité »

24. Les Hauptprobleme der Philosophie sont écrits en 1909 et publiés en 1910. Le premier chapitre, «De l'essence de la philosophie », définit celle-ci comme le fait de «présenter le plus universel dans la forme d'une individualité typique », les chapitres suivants traitent des catégories fondamentales de la philosophie : «De l'être et du devenir » (chap. 2), « Du sujet et de l'objet » (chap. 3), tandis que le dernier, «Des exigences idéales », propose une théorie de la valeur. Les passages que nous citons sont extraits du troisième chapitre. Sur invitation de la G. J. Göschen Verlagsbuchandlung, les Hauptprobleme sont publiés comme cinqcentième numéro de la Sammlung Göschen et seront réédités cinq fois en dix ans. Dès 1912, sous le titre «De l'essence de la philosophie», une grande partie du premier chapitre fut traduite en français (Simmel, Mélanges de philosophie relativiste, Paris, Félix Alcan, trad. fr. A. Guilain). Le reste de l'ouvrage n'a été traduit ni en français ni en anglais.

25. Voir G. Frege, « La pensée » (trad. J. Benoist), dans B. Ambroise et S. Laugier (éds.), Philosophie du langage, Paris, Vrin, 2009, p. 108.

26. $H P$, p. 99.

27. Ibid. 
mais sert aussi la réflexion sur les fondements d'une philosophie de la culture. En résumé, il s'agit de mener à bien un travail de "critique culturelle » tout en essayant de poser les fondements d'une véritable philosophie de la culture, tâche qui requiert une ontologie de l'esprit. La première tâche peut être accomplie en en restant au niveau d'une analyse de l'esprit objectivé et de sa relation avec les individus. La seconde nécessite l'élaboration du concept d'esprit objectif.

Nous conclurons cette première analyse en commentant une phrase qui suit immédiatement l'exposé de l'analogie avec la doctrine des Idées et qui indique une voie permettant d'articuler esprit objectif et esprit objectivé, ontologie et histoire de la culture :

La culture objective est la présentation (Darstellung) historique ou la condensation (Verdichtung) plus ou moins parfaite de cette vérité objectivement valide (sachlich gültigen), dont notre connaissance est une reproduction. ${ }^{28}$

Simmel semble affirmer ici l'existence d'une causalité métaphysique entre l'esprit objectif pensé comme «royaume des Idées» et l'esprit objectivé. L'esprit objectivé serait une sorte de devenir-matériel de l'esprit objectif et l'histoire une réalisation progressive des possibilités de sens recelées par l'esprit objectif, comme si tout ce qui pouvait être pensé tendait à l'être et à prendre une forme sensible, à s'incarner dans les œuvres de l'humanité. Ainsi serait-il d'autant plus pertinent de comparer la culture moderne au royaume des Idées; le cours de l'histoire est l'avènement progressif de ce royaume. Le royaume des idées existe, nous l'avons créé ou plutôt nous l'actualisons sans cesse.

Le rôle accordé à Platon est significatif. Le philosophe de la culture, malgré son vocabulaire teinté de hégélianisme, trouve dans un modèle anhistorique un outil plus efficace que la philosophie de l'histoire de Hegel. C'est que la métaphysique de Platon, telle que Simmel se l'approprie, permet aussi bien, voire mieux que celle de Hegel de penser la densité ontologique des œuvres de l'esprit. Et surtout, elle permet mieux de penser la disproportion entre le sujet et la culture objective et ne porte pas en elle l'espoir illusoire de synthèse et de réconciliation que contient le modèle dialectique. L'analyse des phénomènes d'objectivation et d'autonomisation de l'esprit et de réification de la vie individuelle va faire apparaître plus clairement la nécessité de faire le deuil d'une telle perspective.

\section{$\operatorname{Objectivation(s)}$}

Notre étude requiert à présent une clarification des différents processus d'objectivation présentés dans la Philosophie de l'argent.

28. $P G$, p. 626 (trad. fr. p. 578). 


\section{Le devenir-objet de l'esprit}

Le premier processus correspond à l'allemand Vergegenständlichung. C'est le plus connu et nous ne nous y attarderons pas. Le mot désigne le processus par lequel apparaît de l'esprit objectivé : «avec l'objectivation de l'esprit est donnée la forme qui permet la conservation et l'accumulation du travail mental ». C'est elle qui «offre à l'homme son monde ${ }^{29}$, un ensemble d'objets (Gegenstände) qui portent la marque de l'esprit. Le vocabulaire de la dialectique sujet/objet est tout à fait indiqué dans la mesure où il s'agit de décrire le devenir-objet de quelque chose qui n'a d'abord de réalité que subjective. Simmel n'élabore pas précisément ce concept, il en use de manière non problématique, comme d'un terme permettant de désigner un processus dont on a une idée suffisamment claire. Il ne sert qu'à introduire deux autres processus sur lesquels se concentre l'intérêt de l'auteur.

\section{Un processus d'objectivation immanent à la culture}

Une philosophie dialectique simple de la culture pourrait se satisfaire de ce concept d'objectivation, auquel elle devrait adjoindre une «resubjectivation» permettant la culture individuelle. Ce schéma élémentaire mais éclairant est d'ailleurs utilisé par Simmel au début de la section, lorsqu'il montre comment «la culture des choses [est] culture des hommes $»^{30}$. Mais ce qui intéresse Simmel est l'insuffisance de ce modèle, notamment pour décrire la culture moderne. Avec celle-ci se développe en effet le sentiment que le cours de l'histoire échappe, que les productions du travail deviennent autonomes, ont un sens de moins en moins humain, etc. Pour conceptualiser cela, Simmel introduit un «processus d'objectivation » qui concerne les objets culturels eux-mêmes et qui est immanent à la culture objective. «Le processus d'objectivation (Objektivierungsprozess) des contenus culturels [...] instaure entre le sujet et ses créations une altérité grandissante $»^{31}$. Il ne s'agit pas simplement de désigner la nécessaire altérité entre le sujet créateur et son œuvre. L'altérité dont il est question croît, est susceptible de degrés; c'est de cet accroissement qu'il faut rendre compte.

\section{Une culture objective... de plus en plus objective}

Le contenu culturel devient de plus en plus fortement et consciemment un esprit objectif (objektiv), vis-à-vis non seulement de ceux qui le reçoivent (aufnehmen), mais aussi de ceux qui le produisent. Au fur et à mesure que cette objectivation (Objektivation) progresse, l'étrange phénomène dont nous sommes partis se comprend mieux, à savoir que la croissance culturelle des individus peut prendre un retard sensible par rapport à celle des choses ${ }^{32}$.

29. Ibid., p. 627 (trad. fr. p. 579).

30. Ibid.

31. Ibid., p. 637 (trad. fr. p. 588).

32. Ibid., p. 643 (trad. fr. p. 593). 
Que peut signifier pour un contenu culturel de devenir « de plus en plus [...] esprit objectif »? En quoi celui-ci est-il susceptible de degrés, de plus et de moins ? Il ne s'agit pas seulement de dire qu'il y a de plus en plus d'esprit objectif disponible grâce au travail hérité et conservé. Le fait dont il faut rendre compte est le suivant : le sens des objets culturels pour l'individu est de moins en moins clair. Leur valeur objective est indéniable mais pas leur valeur pour le sujet, ce qu'ils peuvent lui apporter. La qualité objective des objets culturels ne recouvre pas leur «signification culturelle» :

La signification culturelle (Kulturbedeutung) de ses éléments isolés [de culture objective] se mesure pratiquement à leur capacité plus ou moins grande à devenir des facteurs d'évolution (Entwicklungsmomenten) pour les individus eux-mêmes ${ }^{33}$.

Les contenus de culture objective n'ont pas la même valeur selon qu'on les mesure à l'aune de critères objectifs (vérité scientifique, efficacité économique, efficacité technique, etc.) ou en tant que moyens potentiels en vue du développement de l'individu - en l'occurrence par la culture subjective. Une théorie mathématique complexe a une grande valeur objective mais une faible signification culturelle. Pour reprendre les termes du Concept et la tragédie de la culture, nous voyons se développer la «discrépance entre la signification objective (Sachbedeutung) et la signification culturelle ${ }^{34}$ des objets culturels.

Cet accroissement de l'objectivité concerne aussi bien celui qui « reçoit» la culture objective que celui qui la « produit». Chacun est soumis aux normes objectives qui orientent le cours de la culture. Le créateur de culture, quasiment contraint à la spécialisation s'il veut participer aux processus de production, se voit soumis à une logique objective incompatible avec l'idéal de culture subjective. Le processus d'objectivation doit ici être articulé au phénomène de division du travail, qui est son moteur historique principal :

S'il faut condenser en un seul concept ces causes [celles de la discrépance entre culture subjective et culture objective], ce sera celui de la division du travail, pour ce qu'il signifie tant au sein de la production que de la consommation $^{35}$.

La division du travail impose aux producteurs - d'objet techniques comme d'œuvres intellectuelles - d'agir selon une logique purement objective. Elle exige des activités unilatérales et produit des contenus spécialisés dotés d'une faible valeur culturelle, que les individus s'approprient la plupart du temps non pour eux-mêmes mais pour les utiliser dans un processus de production à nouveau spécialisé.

33. Ibid., p. 627 (p. 579).

34. BTK, p. 401 (p. 199).

35. $P G$, p. 628 (p. 580 ). 
Ces analyses sont relativement connues et la division du travail est l'un des objets les mieux traités par la critique culturelle et la sociologie naissante, aussi n'irons-nous pas plus loin. Nous voulons montrer en revanche que Simmel envisage - peut-être sous la forme d'une intuition plutôt que d'une thèse bien établie - un autre type de causalité pour rendre compte du processus d'objectivation. Cette autre causalité rejoint ce que nous avons nommé plus haut une « causalité métaphysique ».

\section{Une logique immanente à l'esprit objectivé ?}

L'idée que la culture objective puisse être une «présentation historique » de l'esprit objectif débordait déjà toute description de l'histoire de la culture en termes exclusivement socio-économiques. En nous appuyant à nouveau sur les Problèmes fondamentaux de la philosophie, nous allons pouvoir reconsidérer cette thèse qui suggère l'existence d'une sorte d'activité propre des contenus spirituels objectifs. À l'occasion d'un exposé sur les philosophies du devenir, alors qu'il traite de Hegel, Simmel introduit le concept d'esprit objectif qu'il reprendra au chapitre suivant. Pourtant, bien que recourant à Hegel, Simmel considère avec beaucoup de liberté la position que le concept occupe à l'intérieur du système hégélien ; pour lui, si Hegel peut nous aider à construire le concept d'esprit objectif, c'est bien moins par sa philosophie de l'esprit que par sa logique. Celle-ci a en effet pour objet «les mouvements dans la forme de l'esprit objectif, les mouvements des contenus dotés de sens ${ }^{36}$. Il n'y a pas de mouvement seulement dans la vie psychique ou dans les phénomènes extérieurs, ou du moins y a-t-il quelque chose qui s'apparente à un mouvement dans la réalité immatérielle des concepts et des significations. Si Platon découvre l'esprit objectif, le Hegel de la Science de la logique en découvre selon Simmel la dynamique, en montrant qu'il y a entre les concepts « un type de leur relation et de leur développement (Entwicklung) [...] qui vaut spirituellement et objectivement $»^{37}$. Autrement dit, il existe une logique immanente qui fait que des contenus spirituels «appellent»d'autres contenus spirituels. Cela n'implique pas, selon Simmel, de penser l'esprit comme un sujet ainsi qu'il reproche à Hegel de l'avoir fait. C'est parce que des individus pensent qu'il y a une vie de l'esprit. Mais ce qu'ils pensent, ils le pensent en partie malgré eux, soumis à une logique que l'on ne saurait réduire à des mécanismes psychiques. Si l'histoire de la culture peut être considérée comme une «présentation historique » de l'esprit objectif, c'est parce que les contenus spirituels «tendent » à engendrer de nouveaux contenus, à condition d'être les objets d'actes psychiques, d'être pris dans des processus vivants qui vont par ailleurs leur donner une forme sensible. Nous ne sommes donc pas contraints de considérer comme toujours déjà donnée la «vérité objectivement valide» dont il était question plus haut et que nous avons 
assimilée à l'esprit objectif. Utilisant Hegel en abandonnant le système et notamment l'esprit absolu -, Simmel évite bien toute perspective de réconciliation du sujet et de l'objet. Par ailleurs, il n'abandonne pas l'analogie platonicienne, si intéressante pour penser la distance du sujet à l'objet: on la retrouve en 1918, dans Lebensanschauung, son dernier ouvrage ${ }^{38}$.

L'articulation des causes historiques et de l'ontologie de l'esprit est fort problématique. Il ne semble pas que Simmel s'y confronte frontalement. Une des principales difficultés est de rendre compte des variations de vitesse des processus historiques. Si les contenus spirituels tendent à en engendrer de nouveaux, pourquoi y a-t-il des moments de stagnation et d'accélération de leur production? Comment articuler causalité idéelle et causalités matérielles ? Nous laisserons ces questions en suspens, pour aborder le processus de réification.

\section{La réification}

À la fin de la section étudiée de la Philosophie de l'argent apparaît un nouvel adjectif attaché à l'esprit : verdinglicht - chosifié ou réifié. Le peu d'occurrences de l'épithète, l'absence du substantif (Verdinglichung) ${ }^{39}$ le manque de thématisation expresse nous imposent une lecture qui prenne le risque de reconstruire et fixer les concepts pour faire droit à la richesse du texte. Citons le passage où l'adjectif apparaît pour la première fois :

L'individu sait-il sa vie intérieure apparentée ou étrangère au mouvement culturel objectif (objektiven Kulturbewegung), ressent-il la supériorité de ce dernier [...] ou ressent-il sa valeur personnelle vis-à-vis de tout esprit réifié (verdinglicht) ? Au sein de sa propre vie spirituelle (Geistesleben), les éléments objectifs, historiquement donnés, représentent-ils une puissance ayant sa propre légalité, si bien que celle-ci et le noyau de sa personnalité se développent indépendamment l'un de l'autre, ou bien l'âme est-elle pour ainsi dire maîtresse de sa propre maison ou du moins établit-elle, entre sa vie la plus intime et ce qu'elle doit y intégrer de contenus impersonnels, une harmonie quant à l'intensité, au sens et au rythme $?^{40}$

Cette première mention n'indique pas en toute clarté la nécessité de donner un sens spécifique à l'adjectif « réifié ». L'esprit réifié dont il est question ici est-il vraiment distinct de l'esprit objectivé ? Nous pouvons cependant noter le contexte où cette expression apparaît pour la première fois. Simmel passe de la relation «extérieure » entre l'individu et la culture objective à une relation «intérieure » qui unit l'individu - ou plutôt

38. Lebensanschauung, in GSG 16, voir par ex. p. 244.

39. Le mot apparaît une fois dans la Philosophie de l'argent, dans un autre contexte, pour désigner le processus par lequel la propriété se détache de la personne, perd sa dimension qualitative, devient abstraite et se fluidifie ( $P G$, p. 366 (trad. fr. p. 336)).

40. $P G$, p. 649 (trad. fr. p. 599, modifiée). 
son «noyau de personnalité ${ }^{41}-$ et les contenus spirituels en lui. Ce déplacement des problèmes vers le plan de la constitution psychique est essentiel: des enjeux anthropologiques majeurs apparaissent en pleine lumière puisque c'est la structure profonde de l'individu qui est présentée comme potentiellement affectée par la tension entre culture objective et culture subjective.

L'écart grandissant entre elles ne signifie cependant pas que l'individu tende à se vider de contenus spirituels, ne s'approprie plus aucun contenu de culture. La question porte plutôt sur le type d'appropriation de ces contenus. La seconde phrase du passage cité suggère deux types d'appropriation. Soit les contenus spirituels se retrouvent dans l'âme en quelque sorte comme ils sont à l'extérieur d'elle, soumis à leur propre légalité, au point que l'âme ne serait qu'une sorte de réceptacle sans effet sur ce qu'elle contient, sans capacité de mise en forme. Soit ils se voient soumis à une forme imposée par un "noyau de la personnalité », assurant l'unité globale des contenus et l'harmonie entre ce qui est personnel et ce qui ne l'est pas d'abord. C'est à partir de ce problème qu'il faut donner sens à l'expression d' « esprit réifié ». La seconde occurrence le confirme : décrivant plus loin le processus par où la vie individuelle devient toujours plus impersonnelle et objective, Simmel désigne le moi comme le «reste non réifiable de celle-ci ${ }^{42}$. Aussi loin qu'aille la soumission de l'âme à des processus impersonnels, il reste toujours en elle un noyau absolument subjectif, c'est-à-dire non réifiable.

Le processus de réification accompagne donc le processus d'objectivation, il en est le versant anthropologique. Mais il faut distinguer les deux processus, dans la mesure où leurs objets sont ontologiquement distincts : le premier est la culture objective, le second l'âme, la vie subjective. Il faut aussi insister sur le fait que cette réification n'est pas une négation de la subjectivité, mais l'échec de la mise en forme des contenus par cette subjectivité. L'âme de l'homme moderne tendrait à se scinder en deux «parties» n'interagissant plus et se développant de manière autonome : les contenus spirituels empruntés à l'esprit objectivé d'un côté, le moi de l'autre. Ce moi peut dès lors s'affirmer plus que jamais, la conscience d'être un sujet se renforce. Mais cette individualité qui s'affirme court le danger d'être formelle, sans matière, dans la mesure où elle peine à imposer sa marque aux contenus spirituels qui l'habitent ou, pourrait-on dire, la parasitent puisqu'ils «profitent» de sa vie pour se développer sans servir en retour les fins proprement individuelles.

41. Cette image du noyau ou du germe de la personnalité apparaîtra de plus en plus souvent chez Simmel, en même temps que se développera son «individualisme qualitatif » d'inspiration romantique. Sur ce point, voir par exemple, en français, «L'individualisme » et «Individualisme et liberté » in G. Simmel, Philosophie de la modernité, Paris, Payot et Rivages, 2004, tr. fr. J-L. Vieillard-Baron (désormais PM). Dans la Philosophie de l'argent l'expression désigne le foyer de la subjectivité, ce qui précisément résiste à toute réification, plutôt que l'être absolument singulier de l'individu, comme ce sera le cas plus tard.

42. Ibid., p. 652 (trad. fr. p. 602) (nicht zu verdinglichende[r] Rest). 
Les processus à l'œuvre dans la culture moderne conduisent donc au durcissement de l'opposition subjectivité/objectivité et rendent caduques les perspectives de synthèse dialectique. En outre la culture objective ne saurait être pensée comme une simple expression et objectivation de la vie (au sens 1) : elle suit un mouvement qui est largement immanent et déterminé par des processus qui, au plan des causalités historiques comme au plan ontologique, se servent de la vie plutôt qu'ils ne l'expriment. Comprendre les contenus de la culture objective n'est donc pas seulement comprendre comment une vie s'y exprime, mais plutôt comment une légalité immanente à l'esprit s'y déploie. Il faut à présent changer d'échelle et voir que le primat de l'objectif sur le subjectif s'impose déjà quasiment dans toute confrontation entre un individu et un objet culturel. C'est ce que montre, chez Simmel, la question du rapport aux œuvres de l'esprit.

\section{Le contenu spirituel est plus riche que toutes ses actualisations individuelles}

\section{L'objectivité du contenu et sa reproduction dans la vie psychique}

Revenons aux Problèmes fondamentaux de la philosophie. Dans un passage qui précède celui commenté plus haut, quelques remarques sur le rapport aux œuvres prennent place dans la discussion sur l'esprit objectif.

Dans l'histoire du genre humain se développe une grande série de formations (Gebilden), qui sont créées par l'invention et le travail psychique mais qui, une fois ce travail accompli, acquièrent une existence singulière et objective (objektiv), au-delà des esprits individuels qui les ont originellement produits ou qui les reproduisent ultérieurement ${ }^{43}$.

La suite du texte montre que l'auteur pense ici particulièrement à ce que l'on a coutume de nommer des œuvres, c'est-à-dire des objets culturels dotés d'une signification propre, relativement close et autonome. Ce passage contient trois thèses importantes : 1 . une fois produite une œuvre existe par elle-même, indépendamment de celui qui l'a produite et de celui qui la reçoit; 2. son contenu ne s'épuise pas dans les actes psychiques qui s'y rapportent, du côté de la production ou de la reproduction ; 3 . la réception de l'œuvre est une « reproduction » de son contenu. La dernière thèse implique qu'un même contenu puisse être présent en différents esprits. Elle est logiquement liée à la thèse précédente : si tout acte psychique est singulier, il est en revanche possible de concevoir le contenu pensé comme intemporel et impersonnel, et de le distinguer soigneusement de ces actes. Prenant l'exemple d'un livre, Simmel continue :

43. HP, p. 67. 
Le contenu que le lecteur forme en lui par un processus vivant est contenu dans le livre sous une forme objective, le lecteur le «prélève » (« entnimmt») du livre. S'il ne prélevait pas ce contenu, le livre ne le perdrait pas pour autant et sa vérité ou sa fausseté, sa noblesse ou sa vulgarité ne dépendent pas du tout du fait que le sens du livre soit rarement ou fréquemment, avec une pleine compréhension ou sans compréhension, reproduit (wiedererzeugt) dans les esprits subjectifs ${ }^{44}$.

À nouveau sont distingués les faits psychiques qui permettent d'appréhender le contenu spirituel et ce contenu lui-même. À nouveau l'appréhension par les individus d'un contenu spirituel à partir d'une formation objective est présentée comme une reproduction. L'idée que le lecteur «prélève » du livre son sens est très frappante. Elle suppose qu'un même contenu de sens puisse être présent dans l'esprit d'individus différents, mais aussi à la fois dans des choses et des individus. Comprendre une œuvre serait procéder à l'extraction d'un sens objectivement présent, de telle sorte que le contenu spirituel sous sa forme objective ou sous sa forme subjective (dans la vie psychique de l'individu) reste inchangé. Cette conception de la compréhension donne à réfléchir. La compréhension renvoie à l'interprétation et, de là, à la question de la singularité de toute compréhension. Or, sur fond d'ontologie réaliste de la signification, Simmel paraît identifier la compréhension réussie à une simple reproduction objective. Nous pouvons réduire cette tension à partir d'un dernier texte de Simmel : De l'essence de la compréhension historique. Le détour par cette question d'herméneutique nous fera appréhender sous une autre perspective le primat de l'objectivité sur la subjectivité.

\section{L'excès irréductible du sens objectif sur le sens compris}

Nous trouvons dans ce texte de 1918 une distinction intéressante entre compréhension historique et compréhension objective. Comprendre historiquement un poème par exemple, c'est reconstituer les processus psychiques qui ont conduit à sa rédaction, savoir quelle femme, quel événement de la vie du poète, a inspiré tel ou tel vers, etc. Or cette compréhension ne concerne pas, selon Simmel, «la qualité propre de son être $[\ldots]$, son contenu et sa signification poétique [...], ses déterminations immanentes ${ }^{45}$. Celles-ci doivent précisément être l'objet d'une compréhension objective qui est «an-historique » (geschichtsfrei) et doit reposer sur les propriétés de l'œuvre, abstraction faite des conditions de sa genèse. Que le contenu soit lié à la genèse ne constitue pas une objection, la question est de considérer le contenu pour lui-même : en fonction de sa consistance interne, de sa signification, de ses rapports esthétiques, etc. ${ }^{46}$.

44. Ibid., p. 68.

45. VWHV, p. 167 (trad. fr. p. 68) (Eigenschaftlichkeit).

46. Sur ce point notre travail devrait être prolongé par une analyse du statut du contenu idéel de l'œuvre et son rapport avec ce qui tombe sous les sens. L'exemple du poème, et plus 
Nous pourrions penser que la compréhension historique, confrontée à la variété des causes et des effets, portant sur un réel indéfini dans l'espace et dans le temps et dont il faut limiter l'étendue, est le type de compréhension par où la richesse de sens du réel se dévoile au maximum. La compréhension objective, surtout si elle est reproduction, parait condamnée à une morne exactitude ou à l'erreur. Pour Simmel toutefois, c'est le contraire qui est vrai. En effet, si la compréhension historique n'est au fond jamais complète en fait, elle peut l'être en droit. Elle porte sur les relations de cause à effet qui lient des événements, elle ne peut tolérer de «pluralité d'interprétations ${ }^{47}$ : les événements se sont enchaînés comme ceci, non comme cela. En revanche la compréhension objective est potentiellement infinie :

ainsi, face à un seul et même contenu objectif (Sachgehalt), on peut tout à fait satisfaire à l'exigence de le comprendre historiquement ; mais on ne pourra jamais satisfaire totalement à celle de le comprendre objectivement avec tout ce qu'il renferme en lui de significations ${ }^{48}$.

Pour reprendre une distinction devenue classique, la compréhension historique est explication plutôt que compréhension. Or une explication peut être définitive ou du moins son insuffisance ne relève que d'un problème de régression dans la chaîne des causes. L'insuffisance de la compréhension objective est en revanche plutôt un problème d'approfondissement : il ne s'agit pas de convoquer de nouveaux faits mais de pénétrer plus profondément le donné. Le sens objectivement présent est, selon Simmel, en excès sur le sens à chaque fois compris, ce qui naturellement sera d'autant plus vrai que l'œuvre sera plus riche. Il est clair que sa pensée est orientée par son expérience de la confrontation avec les grandes œuvres d'art ${ }^{49}$. C'est en effet une expérience fondatrice chez tous ceux qui aiment sincèrement les arts que de sentir que les œuvres recèlent toujours plus de sens que ce qu'ils sauraient en tirer. Simmel ajoute :

une fois qu'un processus créateur a pris la forme de l'esprit objectivé (objektiviert), tous les types de compréhension, et les plus divers, sont également justifiés dans la mesure où chacun d'entre eux est consistant (bündig) pour lui-même, exact, objectivement (sachlich) satisfaisant ${ }^{50}$.

\footnotetext{
encore sans doute celui des autres types d'œuvres d'art, pose cette question avec insistance : on ne saurait réduire le contenu d'une œuvre d'art à un ensemble de propositions, sous peine de détruire précisément ce qui fait art dans l'œuvre.

47. Ibid., p. 169 (trad. fr. p. 72) (Mehrdeutigkeit).

48. Ibid., p. 170 (trad. fr. p.73, modifiée).

49. L'importance de cette expérience est évidente dans la formation intellectuelle de Simmel et dans l'orientation de ses réflexions. On ne compte pas le nombre d'essais portant sur l'art ou sur des artistes, faisant référence à des œuvres précises, et qui témoignent d'une réception enthousiaste des arts, de la peinture notamment. Voir par exemple, en français, «Rodin », dans $P M$ ou les textes du recueil Le cadre et autres essais, Paris, Gallimard (Le Promeneur), 2003
}

50. Ibid., (trad. modifiée). 
Y aurait-il potentiellement autant de compréhensions que d'individus? Cela n'est-il pas contradictoire avec l'idée selon laquelle la compréhension serait reproduction d'un contenu ? Pour rendre cette thèse de la reproduction compatible avec celle de la légitimité des compréhensions les plus diverses, nous devons considérer toute compréhension comme une reproduction partielle du contenu objectif. C'est le seul moyen d'accorder le réalisme sémantique - si l'œuvre a un contenu objectif, la compréhension doit bien consister en une saisie de ce contenu - avec l'intuition d'un excès du sens objectif sur tout ce que les individus peuvent en saisir. Ainsi, si l'on met de côté celles qui sont manifestement fautives ou contradictoires, la diversité des compréhensions ne provient pas seulement de la diversité des points de vue individuels mais de la plénitude de sens des contenus qu'il s'agit de comprendre.

D'une manière générale, s'il y a distorsion entre le sens de l'œuvre et celui qu'on lui attribue, ce n'est pas tant parce qu'une mauvaise compréhension en corromprait le sens - même si cela peut arriver - mais parce que toute compréhension est nécessairement incomplète. La thèse n'est donc pas relativiste : ce qui autorise la multiplicité des interprétations, ce n'est pas l'impossibilité d'entrer en contact avec la réalité, ni un manque de cette réalité, c'est au contraire sa richesse. Du point de vue d'une ontologie de l'esprit, nous pouvons dire qu'il y a plus d'être (d'esprit) dans l'œuvre que dans ce qui est pensé par l'esprit qui la reçoit... ou qui la produit. Ainsi, si nous comparons l'œuvre à une énigme, comme le propose Simmel, nous dirons que le créateur a une clé de l'énigme mais que ces clés sont en nombre « par principe illimité ${ }^{51}$.

Cet excès de sens n'est pas lié à la complexité des chaînes de causalité qui ont permis la genèse de l'œuvre, non plus qu'à notre incapacité à les connaître : ce serait confondre genèse et contenu. C'est par la vertu de l'esprit objectivement contenu dans l'œuvre que celle-ci recèle un sens inépuisable. Simmel mobilise sur ce point le lexique du possible et de sa réalisation: toute interprétation ne « réalise » (verwirklicht) qu'une partie des «possibilités de compréhension reposant virtuellement dans son objectivité $»^{52}$. Cette virtualité n'est pas un néant rempli par l'arbitraire subjectif, elle est le mode d'être objectif de l'esprit. Les remarques faites plus haut sur la logique et la mobilité de l'esprit peuvent contribuer à éclairer ce point, mais il faut avouer qu'il est difficile de poursuivre l'analyse à l'aide des seuls instruments fournis par Simmel. Simmel lui-même butait déjà sur cette difficulté dans la Philosophie de l'argent lorsqu'il écrivait que le contenu spirituel «habite [dans les ouvres] sous une forme potentielle impossible à mieux définir, partant de quoi la conscience individuelle peut l'actualiser ${ }^{53}$. Il ne s'agissait cependant pas alors de s'interroger sur la

51. Ibid., p. 167 (p. 69, trad. modifiée).

52. Ibid., p. 168 (p. 70, traduction modifiée) (in ihrer Objektivität virtuell ruhende Verstehensmöglichkeiten).

53. PG, p. 626 (trad. fr. p. 578). 
relation de l'individu avec un objet culturel mais avec la culture objective en général. Simmel écrivait ainsi, passant d'un problème d'ontologie de l'esprit à un problème de philosophie de la culture proprement dite :

le travail spirituel de la communauté culturelle ainsi condensé (verdichtet) est donc, par rapport à la faculté de vie dans les esprits individuels, ce que la profusion du possible est par rapport à la limitation de la réalite ${ }^{54}$.

L'esprit contenu en toute œuvre excède déjà la part de ce que l'individu peut s'approprier. À plus forte raison les contenus spirituels de la culture objective prise comme un tout sont-ils infiniment loin d'être réalisés dans l'âme des individus. Cependant, ces deux constats qui reposent in fine sur une même ontologie de l'esprit, ne renvoient pas aux mêmes expériences de l'individu ni ne posent les mêmes problèmes au philosophe. L'expérience de la plénitude de sens de l'œuvre est heureuse et stimulante, l'expérience de l'écart grandissant entre les capacités de l'individu et la culture objective est tragique. La Philosophie de l'argent l'indique déjà et cela deviendra un des thèmes de prédilection de Simmel à partir des années 1910.

\section{Remarques conclusives}

La distance de la philosophie de la culture de Simmel avec les modèles dialectique ou de l'objectivation-expression est définitivement établie. Le recouvrement entre sujet et objet est d'une manière générale si peu possible qu'il n'a même pas lieu dans le rapport peut-être le plus intime du sujet à l'objet, celui du créateur à son œuvre. À peine achevée, l'œuvre possède déjà non pas moins de sens, non pas un autre sens, mais infiniment plus de sens que ce que le créateur voulait y mettre.

Dans Le concept et la tragédie de la culture, Simmel emploie l'expression étonnante de "grâce de l'esprit objectif ${ }^{55}$ pour désigner cette qualité qu'a tout contenu spirituel de receler, diffuser, produire de nouveaux contenus. Cette métaphore théologique confirme le réalisme de l'ontologie simmélienne de l'esprit, qui va presque jusqu'à reconnaître une activité spontanée de l'élément spirituel. Nous ne devons cependant pas perdre de vue que cet élément a besoin de la vie pour se mouvoir et trouve en elle son origine avant de s'en détacher ${ }^{56}$. Cette fécondité de l'esprit produit une culture objective toujours plus pleine et parfaite mais de plus en plus stérile au regard des fins du perfectionnement de soi par la culture. La grâce se mue en malédiction, en tragédie. Elle ne peut être retrouvée qu'à la condition

\section{Ibid.}

55. BTK, p. 407 (p. 206) : Gnade(n) des objektiven Geistes.

56. Sur ce point, les développements les plus aboutis se trouvent dans le deuxième chapitre de Lebensanschauung, au titre évocateur : Le tournant vers l'idée. 
que l'individu réussisse à se ménager des rencontres avec des œuvres, à s'extraire momentanément des processus objectifs qui le distraient, le paralysent ou le réifient. 